\title{
Safety and feasibility of robotic surgery in selected ovarian cancer patients undergoing interval debulking surgery
}

\author{
Christos lavazzo, MD, MSc, PhD', loannis D. Gkegkes, MD, PhD² \\ ${ }^{1}$ Department of Gynaecological Oncology, Metaxa Cancer Hospital, Piraeus; ${ }^{2}$ Athens Colorectal Laboratory, Athens, Greece
}

With a great deal of interest, we read the article entitled "Robotic-assisted interval cytoreductive surgery in ovarian cancer: a feasibility study" by Carbajal-Mamani et al. [1]. The study retrospectively examined 12 patients who underwent interval cytoreductive surgery, with complete cytoreduction achieved in $75 \%$ of patients. The main advantages of the approach were minimal blood loss (100 $\mathrm{mL})$, and length of hospital stay (2 days). Two robotic cases with upper abdominal disease required conversion to open laparotomy to achieve optimal cytoreduction. Regarding short-term outcomes, only one patient had a postoperative port-site hernia. No longterm outcomes or outcomes related to safety were presented because of the small median follow-up time (9.5 months).

The INTERNATIONAL MISSION study concluded that minimally invasive techniques could be used in patients with ovarian cancer undergoing interval cytoreductive surgery; however, this approach was feasible only for low-complexity standard cytoreductive procedures [2]. Moreover, a recent meta-analysis revealed that the minimally invasive approach resulted in less estimated blood loss and a shorter hospital stay, but the authors failed to clarify the oncological safety of the technique, and rates of disease recurrence via a sub-analysis based on stage or histologic type [3]. Another meta-analysis showed that complete cytoreduction could be achieved in $74.5 \%$ of patients in the minimally invasive surgery group compared to $53.10 \%$ in the laparotomy group. Questions could be raised regarding potential patient selection bias in the minimally invasive group since some individuals demonstrated a complete clinical response to chemotherapy and lower tumor loads on diagnostic laparoscopy [4].

The well-designed Carbajal-Mamani et al. [1] study is similar and focused on 57 patients with ovarian cancer who underwent robotic interval cytoreductive surgery. Eightytwo percent achieved complete cytoreduction [5]. This study showed that the robotic approach did not adversely affect overall survival. The median survival in the pre-robotic era was 37.9 months versus 42.8 months in the robotic era. Progression-free survival was 11.9 months in the pre-robotic era versus 16.5 months in the robotic era group. The conversion rate was $10.5 \%$ and no port-site metastases were described.

Traditionally, debulking surgery is performed via laparotomy. However, patients with a complete response to neoadjuvant chemotherapy may achieve complete cytoreduction with less-invasive surgery and may, therefore, be selected for minimally invasive techniques. We agree that complete cytoreductive surgery, using a robotic approach, is safe and feasible in these patients when performed by highly trained gynecological oncologists in selected tertiary care centers. However, concerns that require further clarification may include intra-operative spillage, port-site metastases, suboptimal cytoreduction in cases of upper abdominal disease, and the adequacy of lymph node dissection, bowel surgery,

Received: 2020.03.30. Accepted: 2020.05.17.

Corresponding author: Christos Iavazzo, MD, MSc, PhD

Department of Gynaecological Oncology, Metaxa Cancer Hospital, 51, Botassi Street, Piraeus 18537, Greece

E-mail: christosiavazzo@hotmail.com

https://orcid.org/0000-0003-2119-4599

Articles published in Obstet Gynecol Sci are open-access, distributed under the terms of the Creative Commons Attribution Non-Commercial License (http://creativecommons. org/licenses/by-nc/3.0/) which permits unrestricted non-commercial use, distribution, and reproduction in any medium, provided the original work is properly cited.

Copyright $\odot 2020$ Korean Society of Obstetrics and Gynecology 


\section{Obstetrics \& Gynecology Science}

Vol. 63, No. 5, 2020

diaphragmatic stripping, splenectomy, or widespread upper abdominal surgery.

Based on the encouraging data of the above-mentioned studies, well-powered multicenter randomized trials should be considered. Such trials would overcome the limitations inherent to retrospective single-center findings. However, based on the recent negative results of Laparoscopic Approach to Cervical Cancer (LACC) trial, it is questionable if and when the Gynecological Oncology Society would proceed with such an effort.

\section{Conflict of interest}

No potential conflict of interest relevant to this article was reported.

\section{References}

1. Carbajal-Mamani SL, Schweer D, Markham MJ, Esnakula
AK, Grajo JR, Castagno JC, et al. Robotic-assisted interval cytoreductive surgery in ovarian cancer: a feasibility study. Obstet Gynecol Sci 2020;63:150-7.

2. Fagotti A, Gueli Alletti S, Corrado G, Cola E, Vizza E, Vieira $\mathrm{M}$, et al. The INTERNATIONAL MISSION study: minimally invasive surgery in ovarian neoplasms after neoadjuvant chemotherapy. Int J Gynecol Cancer. 2019;29:5-9.

3. Shi C, Gao Y, Yang Y, Zhang L, Yu J, Zhang T. Comparison of efficacy of robotic surgery, laparoscopy, and laparotomy in the treatment of ovarian cancer: a metaanalysis. World J Surg Oncol 2019;17:162.

4. Cardenas-Goicoechea J, Wang Y, McGorray S, Saleem MD, Carbajal Mamani SL, Pomputius AF, et al. Minimally invasive interval cytoreductive surgery in ovarian cancer: systematic review and meta-analysis. J Robot Surg 2019;13:23-33.

5. Abitbol J, Gotlieb W, Zeng Z, Ramanakumar A, Kessous $R$, Kogan $L$, et al. Incorporating robotic surgery into the management of ovarian cancer after neoadjuvant chemotherapy. Int J Gynecol Cancer 2019;29:1341-7. 